\title{
Using Rule Base System in Mobile Platform to Build Alert System for Evacuation and Guidance
}

\author{
Maysoon Fouad Abulkhair \\ Department of Information Technology \\ Faculty of Computing and Information Technology \\ King Abdulaziz University \\ Jeddah, Saudi Arabia
}

\author{
Lamiaa Fattouh Ibrahim \\ Department of Information Technology \\ Faculty of Computing and Information Technology \\ King Abdulaziz University \\ Jeddah, Saudi Arabia \\ Department of Computer Science and Information \\ Institute of Statistical Studies and Research \\ Cairo University, Cairo, Egypt
}

\begin{abstract}
The last few years have witnessed the widespread use of mobile technology. Billions of citizens around the world own smartphones, which they use for both personal and business applications. Thus, technologies will minimize the risk of losing people's lives. Mobile platform is one of the most popular platform technologies utilized on a wide scale and accessible to a high number of people. There has been a huge increase in natural and manmade disasters in the last few years. Such disasters can happen anytime and anywhere causing major damage to people and property. The environment affluence and the failure of people to go to other safe places are the results of catastrophic events recently in Jeddah city. Flood causes the sinking and destruction of homes and private properties. Thus, this paper describes a system that can help in determining the affected properties, evacuating them, and providing a proper guidance to the registered users in the system. This system notifies mobile phone users by sending guidance messages and sound alerts, in a real-time when disasters (fires, floods) hit. Warnings and tips are received on the mobile user to teach him/her how to react before, during, and after the disaster. Provide a mobile application using GPS to determine the user location and guide the user for the best way with the aid of rule-based system that built through the interview with the Experts domains. Moreover, the user will receive Google map updates for any added information. This system consists of two subsystems: the first helps students in our university to evacuate during a catastrophe and the second aids all people in the city. Due to all these features, the system can access the required information at the needed time.
\end{abstract}

Keywords—safety; Natural disasters; smartphone; rulebased system; Mobile Network; Smart Phone

\section{INTRODUCTION}

Damages and ripple effects can happen due to disasters without notification anytime and anywhere [1]. Disaster break presents many unique logistics challenges, such as damage to transportation infrastructure, limited communication, and coordination of multiple agents [2]. To build a solid disaster management system, three fundamental things need to be addressed [3]: cation.

a) To prevent disaster, we must have strong communi-

b) Mobile user location and real-time pictures of events are very important to make decisions. c) Effective analysis and reasoning engines help in the prediction model, and reduce, and prevent disasters.

Knowledge and personal expertise is represented in the form of rule-based systems that has the form of IF-Then rules. A number of applications are suitable to use Rule-based systems [4], [5] and [6]. New types of spatial computing applications and technologies use GPS and sensors installed on smartphones and other powerful smart devices [7] and [8].

In this paper, we use Rule-based systems, smartphones equipped with GPS and other technology to make our system. Our system is divided into two subsystems:

1) Calamity communicator (iCalamityGuide) will deliver the following in terms of Hardware and Software:

- Build a communicator mobile phone application that broadcasts notification, guiding and giving correct directions as exits from buildings and nearest safe building from an authorized source to guide and locate registered people (students and employees of King $\mathrm{Ab}$ dulaziz University, Jeddah city ) by using WIFI or 3G, and GPS locator.

- Engaging the locating services such as GPS and maps reflecting King Abdulaziz University.

- Establishing a connection using PHP to integrate a double communication link between the database at the hosting server with the iPhone application and XML files. We have the ability to edit this database and reflect the changes on user request from the application.

2) This subsystem is called Disaster smart road guidance DSRG. It solves the problem of real-time disaster and road constructions information. This system notifies the users with the updated information concerning fires, floods, and constructions in Jeddah city in Saudi Arabia. The users can also receive messages about the action they must take before, during, and after a disaster, on their mobile phones. This application provides the users with nearest short path safe location using GPS technology.

Moreover, this application sends Google map updates for any newly added information to the users. Feedback action is also sent to users in a time of trouble using Rule-based system 
feature upon a specific situation. The interview with the Experts domains was the aid in building the Rule-based system. [8].

The rest of this paper is organized as follows. Section 2 discusses the related work. Section 3 introduces the iCalamityGuide and DSRG system. Comparison of related work is done in Section 4. The paper conclusion is presented in Section 5 .

\section{RELATED WORK}

GAIA GPS (FOR HAITIAN DISASTER RELIEF) [9]: The application used to download the map when an earthquake occurs in the area. Up-to-date overlays of disaster sites, hospitals, and other relevant waypoints showed. The application also records GPS tracks, waypoints, and geo-tagged photos. One can also import /export GPX tracks photos and guidance to waypoints and along tracks.

UBALERT- DisASTER ALERT NeTWORK [10]: This application supports interactive map, shoots photos for the disaster, and reports the events with more descriptions. It also sorts events according to severity, location, and popularity.

DISASTER ALERT [11]: This application detects the most popular types of natural disaster from their occurrence time, and it announces the same to all citizens in the same region. The Pacific Disaster Centre (PDC) organization is the main source of the application information. This application presents its information on an interactive user map using global disaster info [11].

In [12], system works on the principles of the client-Server system, wherein the server responds to the requests of the Clients. This system is implemented in two parts: the EMS Client Application, Rescue Application and Server. The Client and Rescue Application are implemented on an Android Application. The server is implemented as a web-based Java Application. This web application is useful for generating area wise issues report, priority wise issues report, and location wise issues report.

\section{DESCRIPTION OF THE SYSTEM}

When a calamity strikes, warning alerts become very important. The existence of some social networking apps as message boards to convey information, apps for receiving news updates, and breaking news help people become aware of a situation.

The process of calamity management involves four phases: mitigation, preparedness, response, and recovery. The mitigation phase attempts to reduce calamity risks by focusing on long-term measures of eliminating calamity. The preparedness phase is the development of an action plan for an upcoming calamity. The response phase is the mobilization of services and relief when calamity strikes and the recovery phase is the restoration of the affected area to its previous state.

\section{A. Implementation of iPhone Calamity Guide iCalamityGuide $i C G$}

This system is built to serve merely the students, staff, employees, and visitors of King Abdulaziz University. The goals of this system are:

- To make the university a safe place for all.

- To make the evacuation plan clear. Guiding a large number of students during the calamity is not an easy task. There are so many blockage problems because they do not know how to act during the calamity.

- To enable the security employees to check and detect the safe places for students and view them on a KAU map.

Figure 1 presents the architecture of the entire system of iCalamityGuide. This system expresses flow and relation through systematic division such as user, interface, web server, and database server, modules for interfaces and service.

In calamity information management, geographic locations are important. Using their mobile phones, they can be located using the mobile network system or using an integrated GPS included in their phone.

GPS can:

- locate,

- calculate the distance traveled,

- record the user's path as a set of waypoints,

- navigate routes,

- work as a compass,

- indicate the elevation above sea level,

- provide the accurate time.

\section{Xcode}

Xcode is an Integrated Development Environment (IDE) containing a suite of software development tools developed by Apple for developing software for OS X and iOS".

It was released in 2003; the latest stable release is version 4.3.2. The main application of the suite called Xcode. The Xcode suite also includes most of Apple's developer documentation, and built-in Interface Builder, an application used to construct user graphical interfaces. It includes modified version of the GNU Compiler Collection as well as, in Xcode 3.1 and later. 


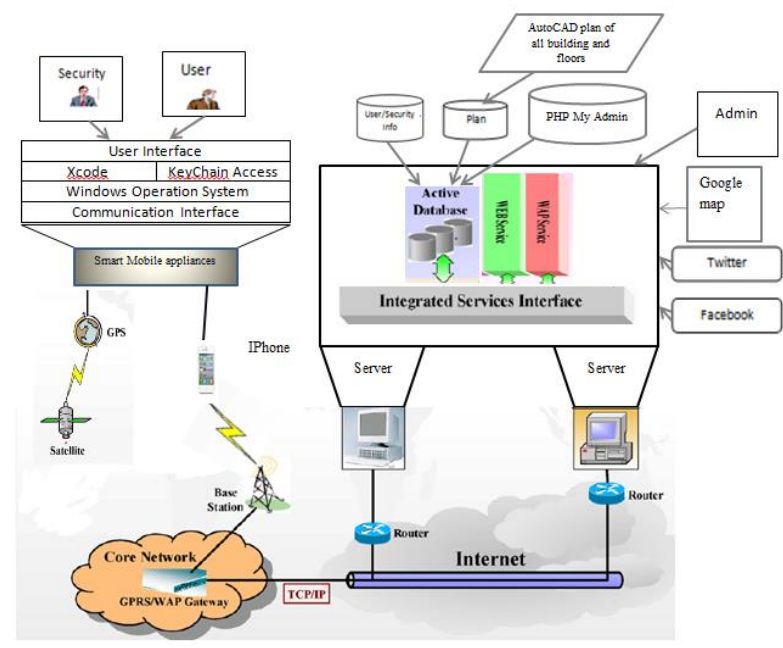

Fig. 1. iCalamityGuide Architecture

"Apple's LLVM-GCC compiler, with front ends of the GNU Compiler Collection and a code generator based on LLVM, and, in Xcode 3.2 and later, Apple's LLVM Compiler, with the clang front end and a code generator based on LLVM, and the Clang Static Analyzer. It supports C, C++, Objective-C, Objective-C++, Java, AppleScript, Python and Ruby source code with a variety of programming models, including but not limited to Cocoa, Carbon, and Java. Third parties have added support for GNU Pascal, Free Pascal, Ada, C\#, Perl, and D. The Xcode suite used the GNU Debugger as the back-end for its debugger. As of version 4.2, the Apple LLVM Compiler became the default compiler. LLDB became the default debugger as of Xcode 4.3".

\section{Keychain Access}

Keychain Access is a Mac application that allows the user to access the Apple Keychain and configure its passwords for Websites, FTP servers, SSH accounts, network shares, wireless networks, groupware applications, encrypted disk images, etc. We used it to gain the keys required for obtaining certificates for Apple developer program to test the application on a real device, as well as distribute it through the Apple store after obtaining the approval of Apple Inc.

\section{PHP My Admin}

"Is a free and open source tool written in PHP intended to handle the administration of MySQL with the use of a Web browser? It can perform various tasks such as creating, modifying or deleting databases, tables, fields or rows; executing SQL statements, or managing users and permissions."'[14]

\section{Features:}

"Features provided by the program include:

1) Web interface

2) MySQL database management

3) Import data from CSV and SQL.

4) Export data to various formats: CSV, SQL, XML, PDF (via the TCPDF library), ISO/IEC 26300 - Open Document Text and Spreadsheet, Word, Excel, LaTeX and others.

5) Administering multiple servers
6) Creating PDF graphics of the database layout

7) Creating complex queries using Query-by-example (QBE)

8) Searching globally in a database or a subset of it

9) Transforming stored data into any format using a set of predefined functions, like displaying BLOB-data as image or download-link.

10) Active query monitor (Processes).”[14]

\section{AutoCAD}

"Is a software application for CAD computer aided design and drafting, the software supports both $2 \mathrm{D}$ and $3 \mathrm{D}$ formats.'[15]

The following section lists the functions used in our system.

\section{- Push notification:}

The application will consciously check if any broadcasts were newely added to the database, and will display if it found an Alert message. The user can then take two actions: either View, which will take them to the application, or cancel.

\section{- Guide me:}

This function describes acquiring guidance where to go. It will display a brief message saying, "Go to Building ...." and the appropriate building based on what arranged from the safety center filled in the database.

This message itself will give two options. The Ok option will zoom out to the campus map as a whole and display the safe destination annotation with a bubble on the top with the tagged message pointing to the safe distention. The second option is Show Exits, which will provide exits for the current building and guidance for the way out in addition to the safe distention.

\section{- Share on Twitter:}

Connectivity to Twitter allows the User to share a picture or tweet so others can share their locations; any helpful information might help others in the same situation.

- KAU:

KAU is an action representing the zooming area that includes KAU Campus. Whenever a user goes away from that area, by this action he/she can get the view back to where it was at the beginning.

- View Safe buildings:

As a Security member, it can display all safe buildings users must go to and it helps to memorize them and keep up with any changes done.

\section{- Update Safe buildings:}

As a Security member, it can update the building's status. In the case of an emergency situation caused to any of the buildings and make them unsafe to stay in and need to be evacuated. 


\section{- Detect users:}

For a Security member, the locations of users can be very helpful to rescue them or make sure they are at a safe building and away from danger, or react toward any crowded locations and take further action to make them safe.

When calamity occurs, the Admin starts the system by sending broadcasting warning news to security employees and users (students, staff, employs and visitors of KAU). Figure 2 (a) shows admin broadcasting an evacuation message to users received as an Alert.

Users respond to the system by push button "View". Then the system starts to detect the location of the user using the GPS location detection. This is followed by a message that represents the evacuation plan regarding that specific spot. Figure 2 shows the sequence in order of screens as viewed by a user. Figure 3 shows sequence in order of screens as viewed by security employees.

d) Implementation of DSRG Disaster Smart Road Guidance

This section will introduce the tools, technologies, and languages used to develop Disaster smart road guidance DSRG package.

\section{Eb2a free Hosting [16]:}

$\mathrm{An} \mathrm{Eb} 2 \mathrm{a}$ is a free hosting site that aids the user during building websites.

\section{Types of panels used by Eb2a [17]:}

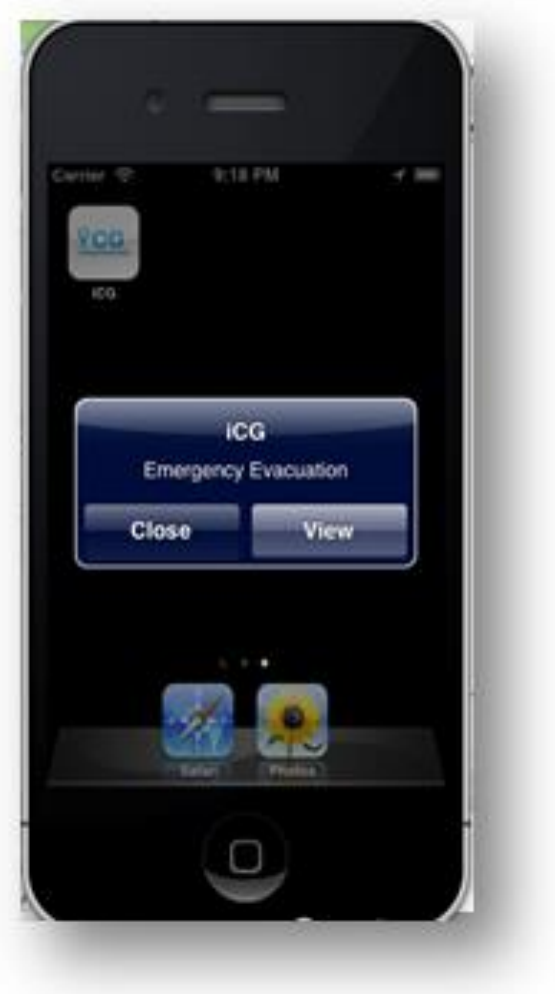

a) Admin sends broadcast message alert
CPanel (Vista panel): is an excellent hosting control panel on the net.

Fantastico: installs complex forums and website templates in an easy way.

Htaccess: our system uses the Htaccess features.

\section{Eb2a features and offers [18]:}

Eb2a servers use the latest versions of PHP, MySQL, and Apache Web Server.

\section{Eclipse SDK platform:}

To create our Android application, we use three key items in the project's root directory [19]:

- AndroidManifest.xml

- Category [20].

- A launcher icon [21].

Rule-based System: A Rule-based is used to collect information. Types of rule and knowledge in the system:

- Domain knowledge.

- Meta-knowledge.

- Common sense knowledge.

- Heuristic knowledge.

- Tacit knowledge.

- Explicit knowledge.

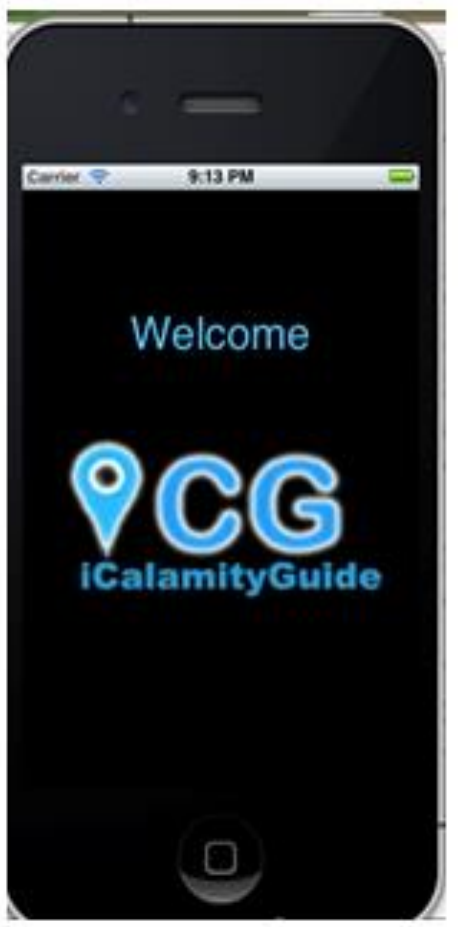

b) Welcome page 


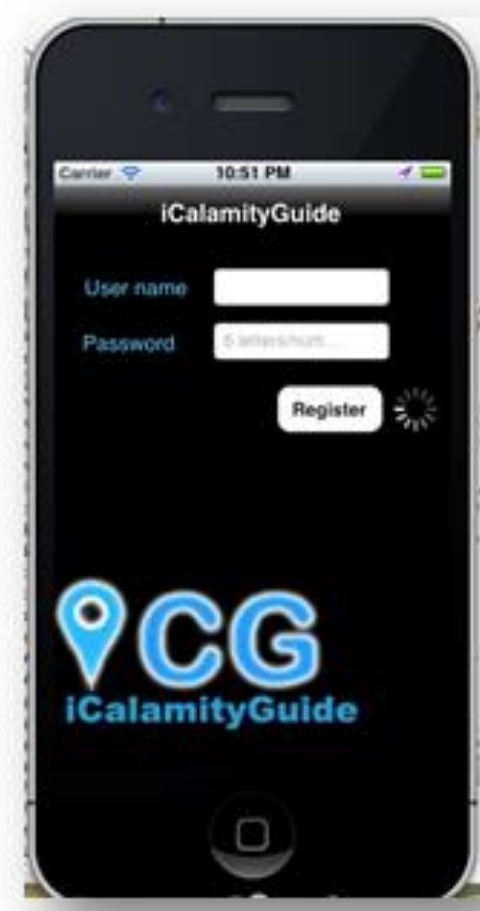

c) iCG user registration

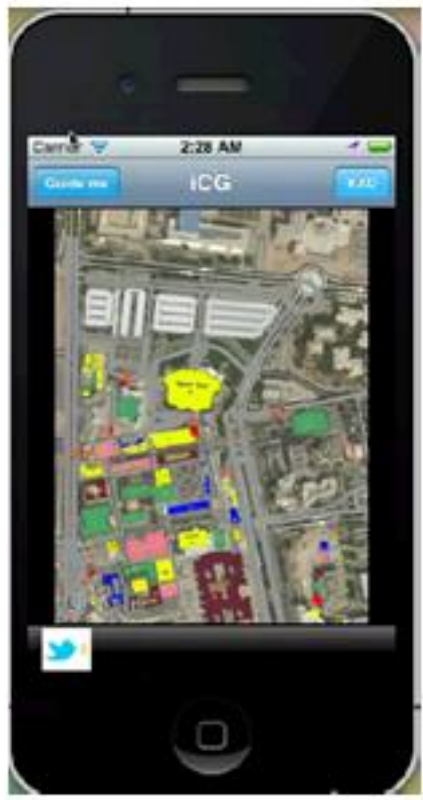

e) After zooming in

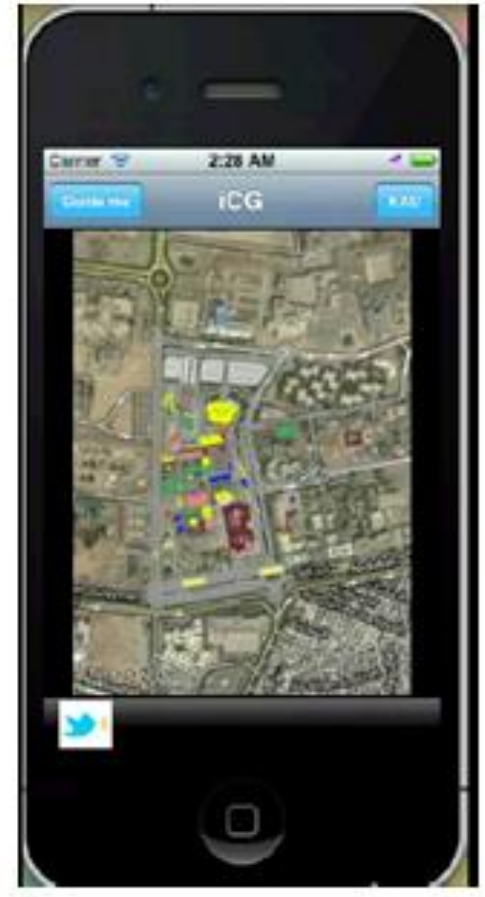

d) The first view for a user

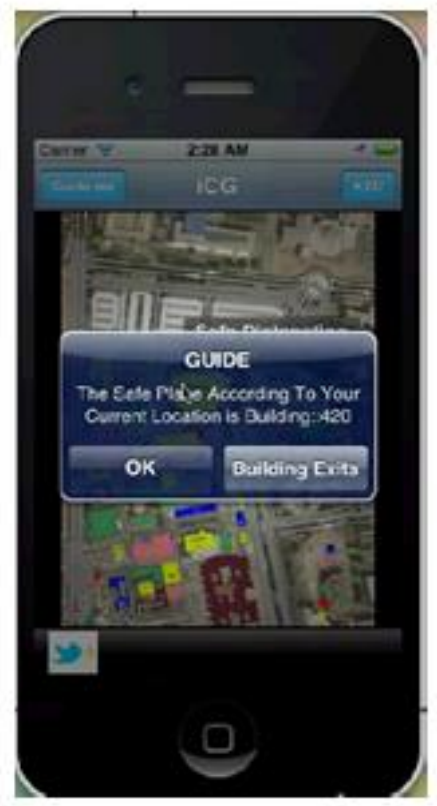

f) the beginning of the guiding screen starts by tapping the Guide me button at top left corner 

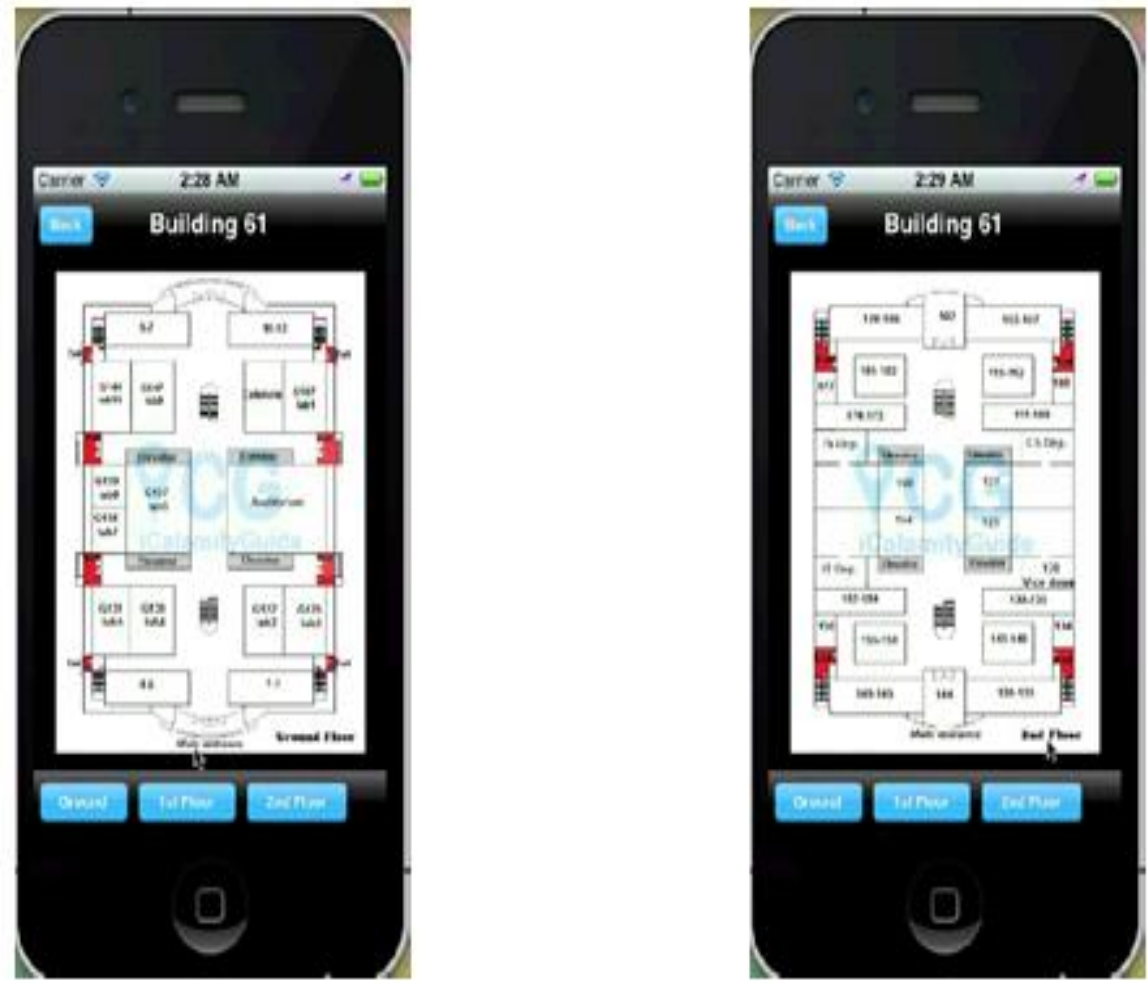

g) After choosing any of the KAU buildings iCG shows the selected floor (First-floor)

h) Second-floor

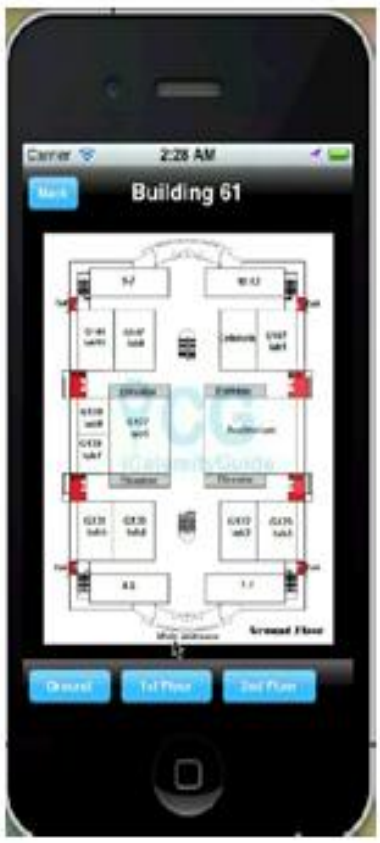

i) Third-floor

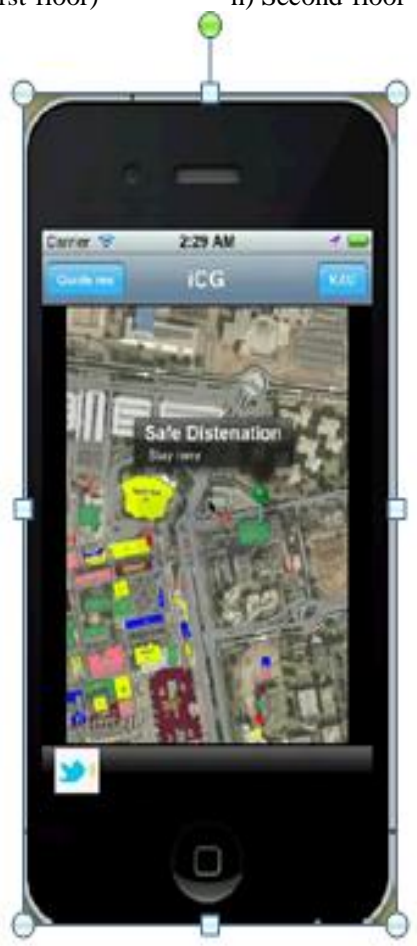

j) Show the user safe destination to go into wherever u goes the bubble will go with $\mathrm{u}$ 


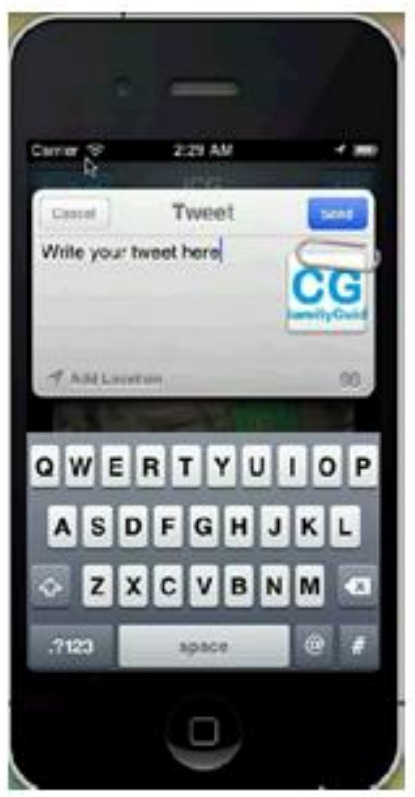

k) After twitter button has been chosen

Fig. 2. Screen sequence User View
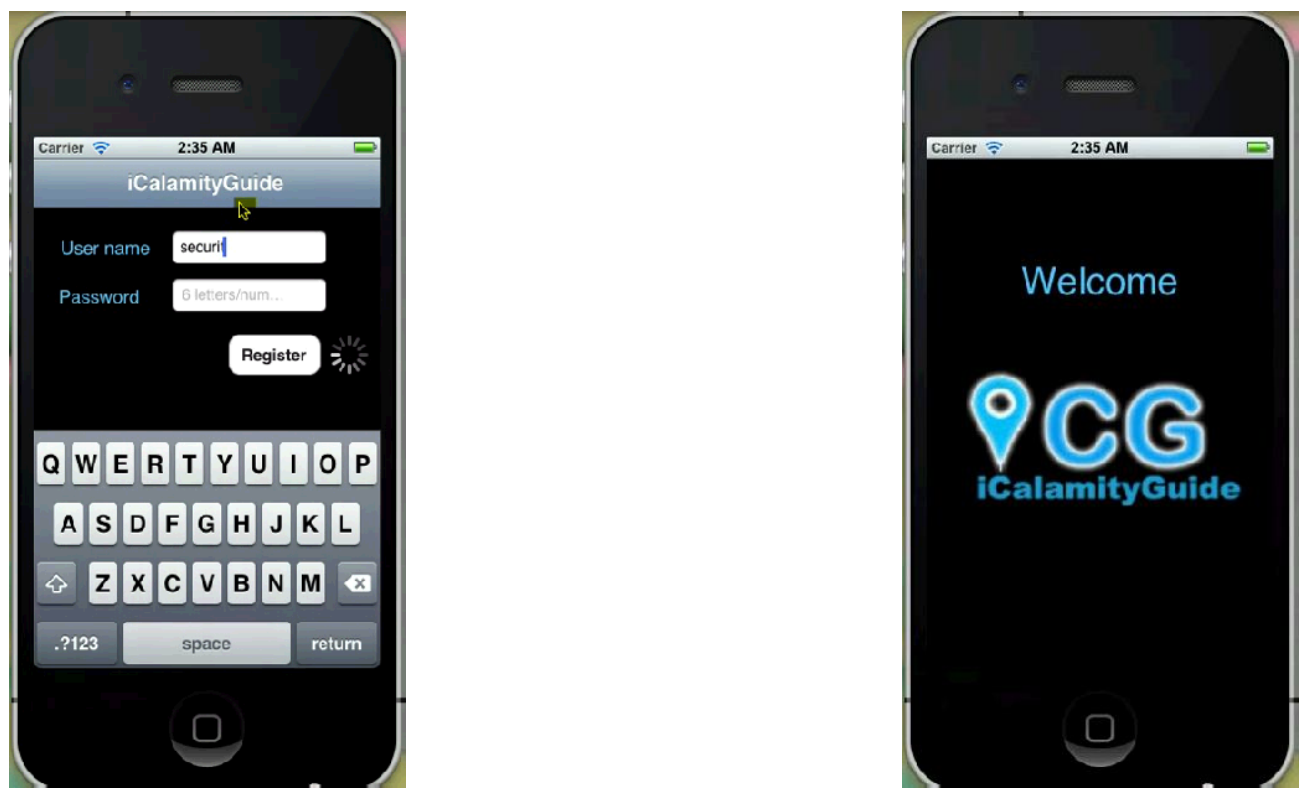

a) Enter the user name and password to recognize if the user is security or regular user

b) Welcome page 


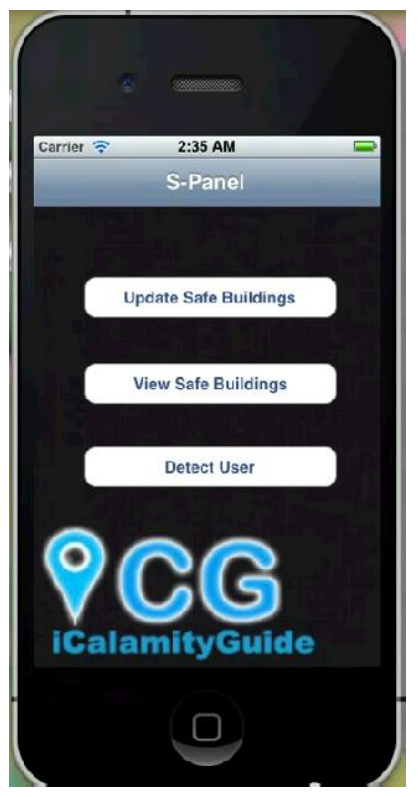

c) Security panel to list the three functions that security can apply

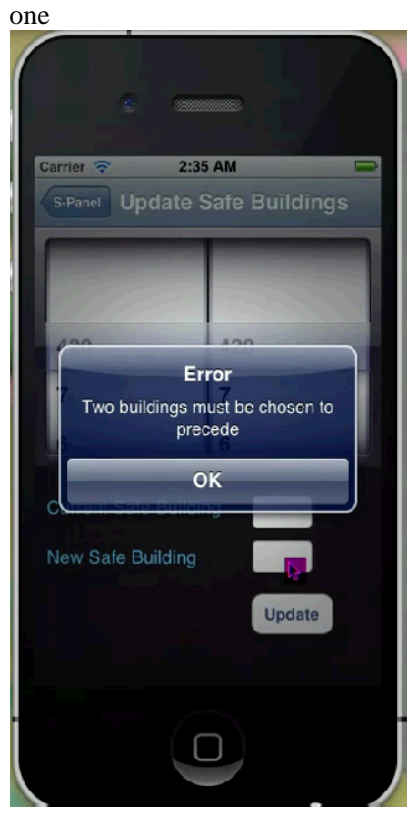

e) Appear if the text field was empty

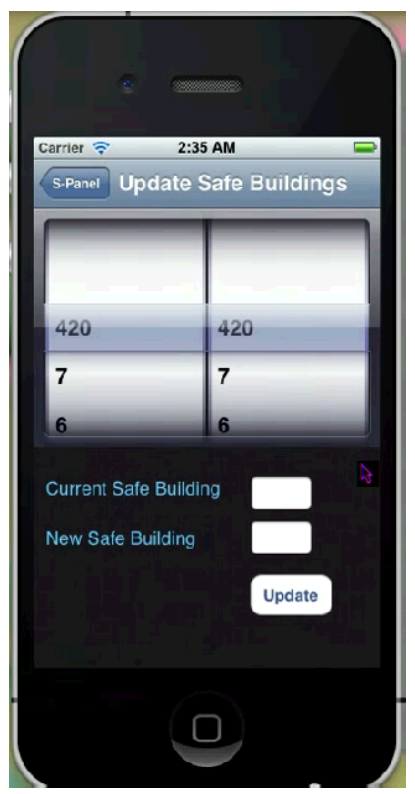

d) After tapping $t$ first button (update the safe building) to replace the unsafe with safe

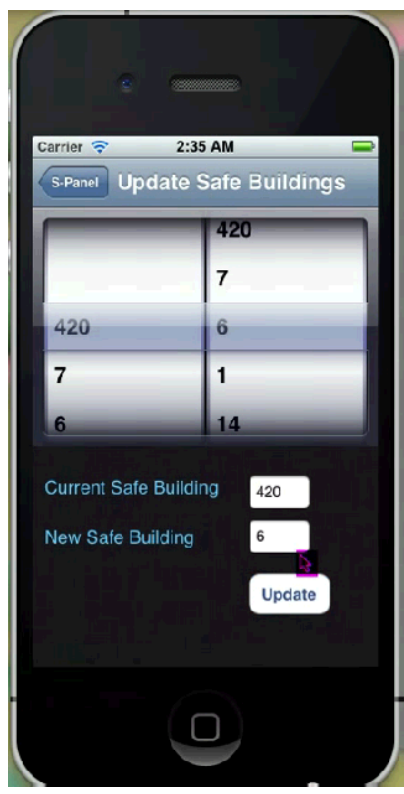

f) The user can scroll the tow lists to fill out all 


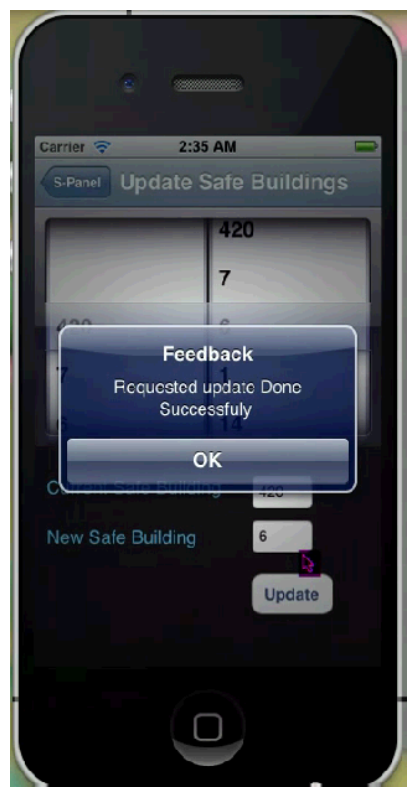

g) Feedback to tell u it has been successfully

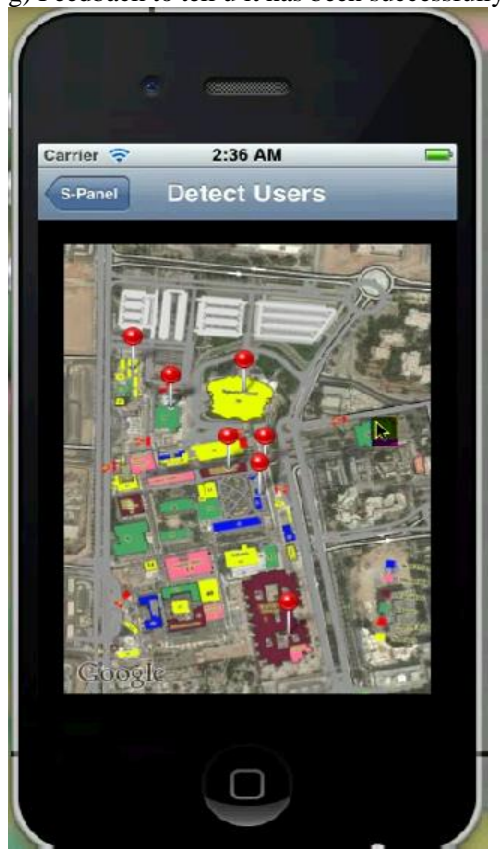

i) The view when selecting the third button that views all pins in map representing all the registered users and their location in the same time

Fig. 3. Screen sequence Security View

Components for DSRG Application Implementation Process are described in Figure 4. As shown in Figure 5, the communication between server and Android is through the database. DSRG application operates on Android mobile phones. Figure 6 shows Android application structure.

Figure 7 shows Home Page and Figure 8 shows Map Screenshot of DSRG.

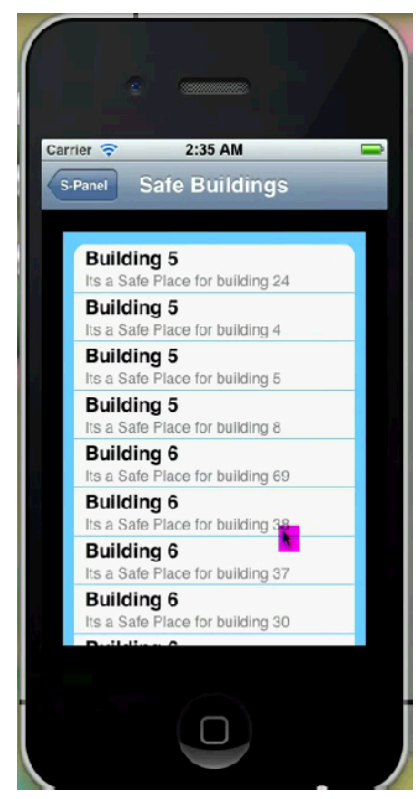

h) Appear when the second button is chosen

\section{COMPARISON OF ICG, DSRG AND OTHER SYSTEMS}

Table 1 compares our systems with two other systems implemented on Android Operating System. iCG is the system used in the indoor environment. iCG and DSRG guide users have the highest reliability. iCG and DSRG use 3G and Wi-Fi in addition to the mobile network. 


\section{CONCLUSION}

A disaster is an unexpected occurrence that happens anytime and anywhere. Most students and people are unaware about the prevention or safety responsiveness to face the disaster. When it happens, an evacuation process is conducted to save the victim. The Mobile platform Alerts Systems iCG and DSRG use the Rule-based system to detect evacuation places guidance mobile application, downloadable and ready to use by regular end users. It incorporates GPS technologies to location specific information and fed it to the citizens providing them with the nearest safe location and the shortest path to get there. It provides the user with evacuation tips depending on the current location and as provided from civil protection agency, weather status updates directly from the PME (Presi-

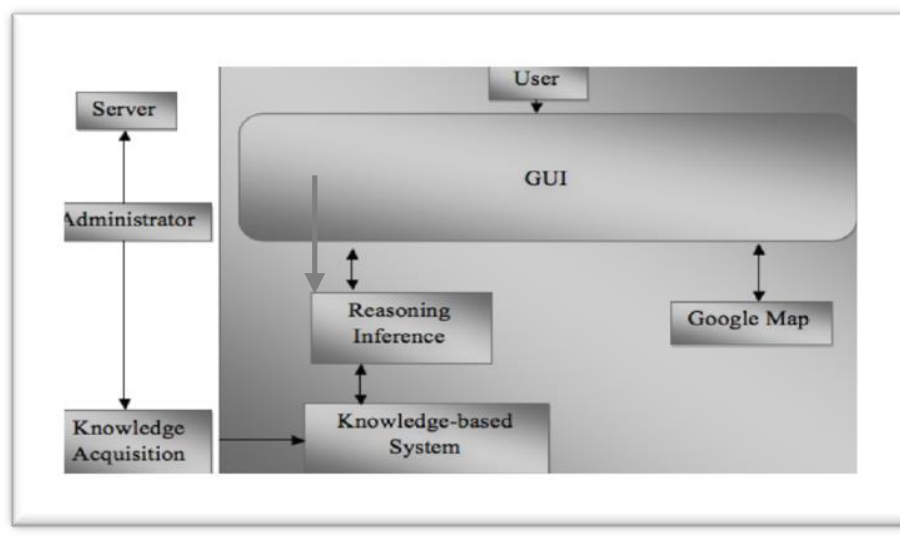

Fig. 4. Components for DSRG System dency of Meteorology and Environment) servers and map services.

This work also delivers real-time disaster and road constructions information and notification to the users such as fires, floods, constructions. Users receive warnings and tips on how to react before, during and after the disaster, directly on their mobile phones. Google map updates for any newly added information is received on the mobile user. In the time of a trouble, the user can benefit from the Rule-based system feature that will give him/her a feedback on what action should take in a specific situation. The Rule-based system was built through an interview with the experts domains. All these features, GPS, Google map, Wi-Fi, 3G and rule-based system, are improved access to the needed information at the needed time to save the life of people and students.

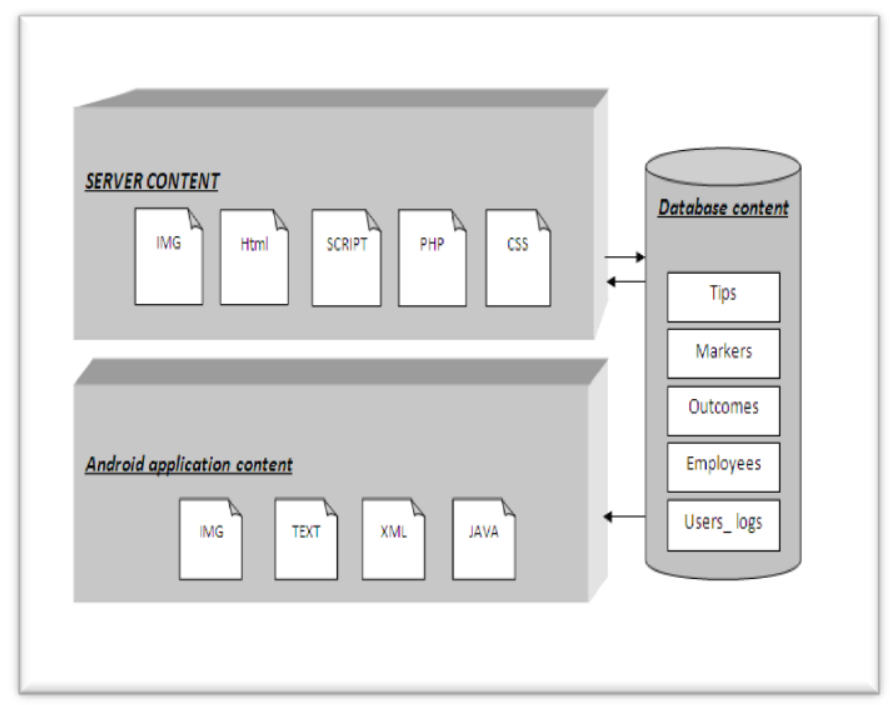

Fig. 5. DSRG Server and Android application content

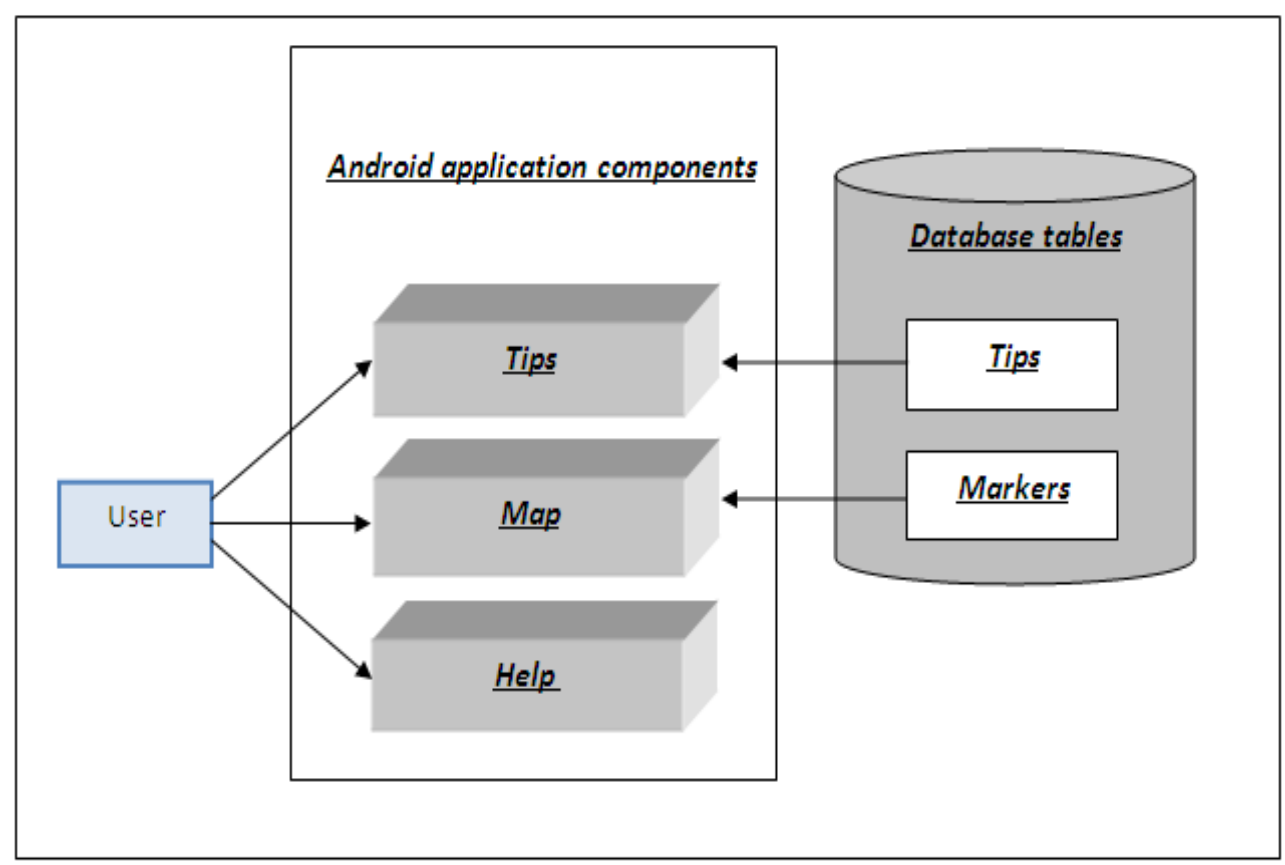

Fig. 6. DSRG Android application structures 


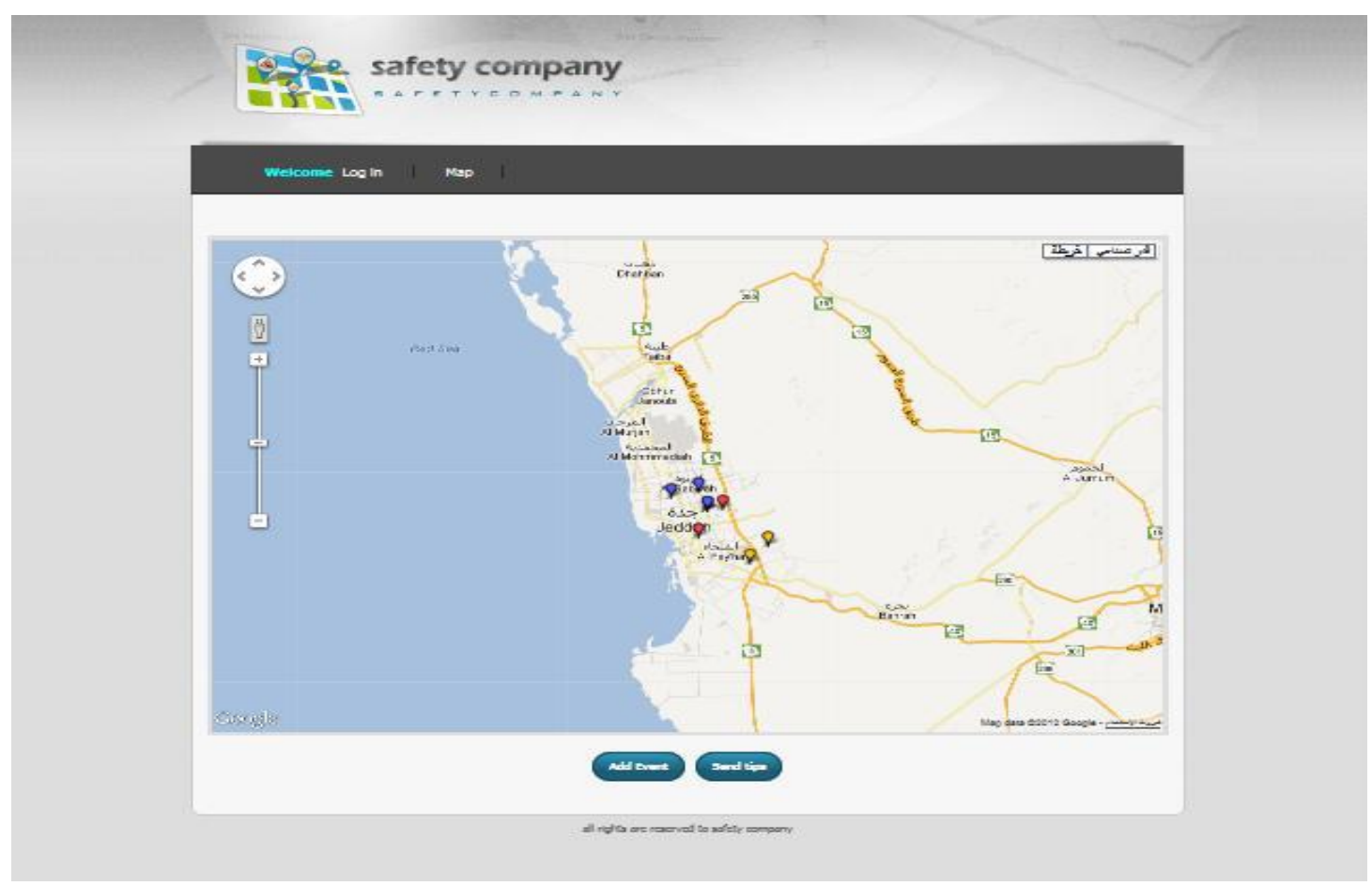

Fig. 7. DSRG Home Page

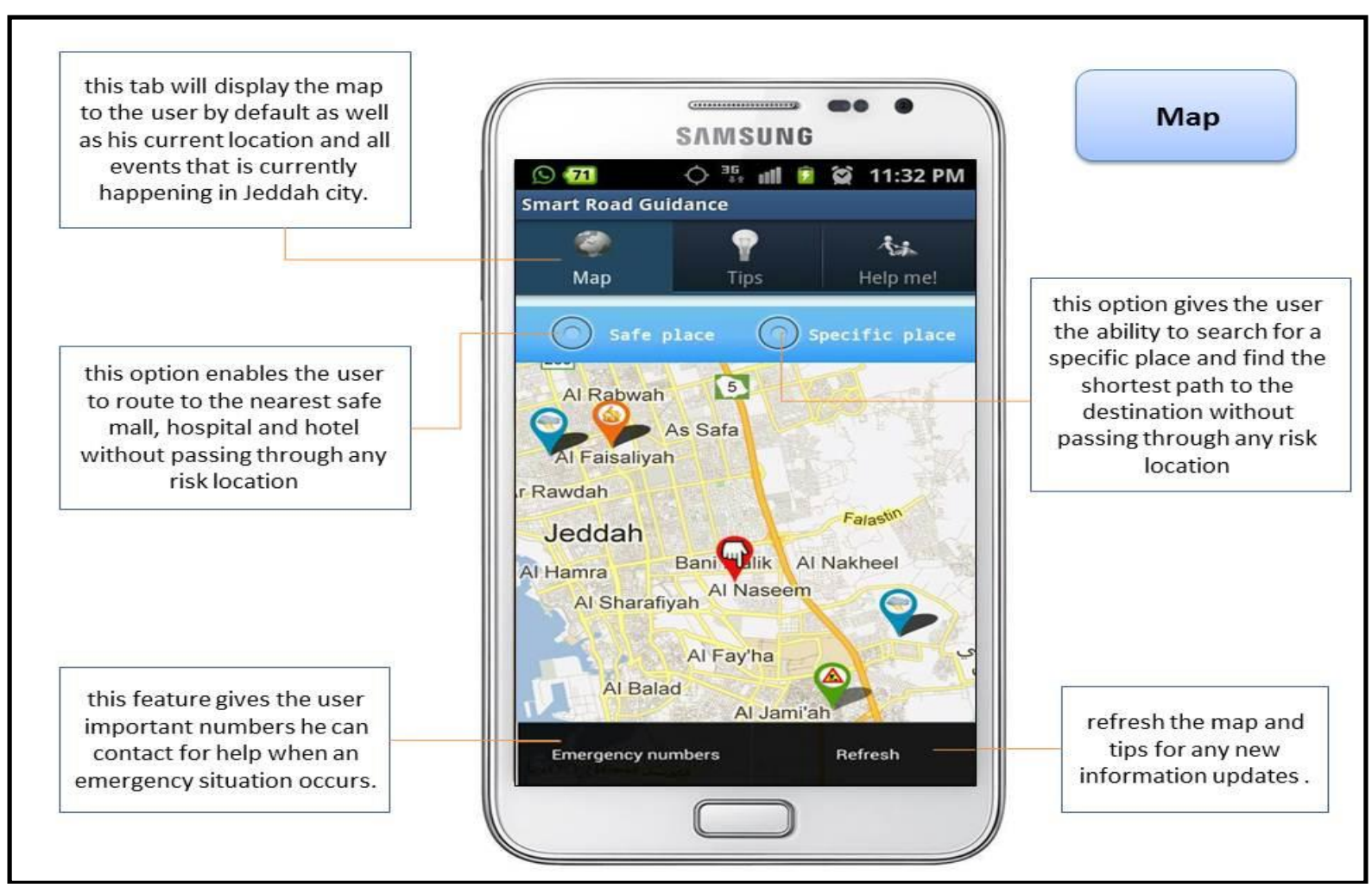

Fig. 8. DSRG Map Screenshot

\section{ACKNOWLEDGMENT}

This work was funded by the Deanship of Scientific Research (DSR), King Abdulaziz University, Jeddah, under grant no. (611-022-D1434). The authors, therefore, acknowledge with thanks, DSR technical and financial support.

\section{REFERENCES}

[1] Hyokyung Chang, Yongho Kang, Hyosik Ahn, Changbok Jang, Euiin Choi, "Context-aware Mobile Platform for Intellectual Disaster Alerts System”, Energy Procedia, Volume 16, Part B, 2012, Pages 1318-1323. 
[2] Luis E. de la Torre, Irina S. Dolinskaya and Karen R. Smilowitz, "Disaster relief routing: Integrating research and practice", Socio-Economic Planning Sciences, Volume 46, Issue 1, March 2012, Pages 88-97.

[3] Hsu-Yang Kung, Hao-Hsiang Ku, Che-I Wu, Ching-Yu Lin, "Intelligent and situation-aware pervasive system to support debris-flow disaster prediction and alerting in Taiwan", Journal of Network and Computer Applications Volume 31, Issue 1, January 2008, Pages 1-18.

[4] J.A. Bernard, "Use of a rule-based system for process control" IEEE Control Syst. Mag., Volume 8, Issue 5, October 1988, Pages 3-13.

[5] M. Nilashi, O. Ibrahim, "A model for detecting customer level intentions to purchase in B2C websites using TOPSIS and fuzzy logic rule-based system” Arab. J. Sci. Eng., 39 (3) (2013), pp. 1-16.

[6] G. Shobha, J. Gubbi, K.S. Raghavan, L.K. Kaushik, M. Palaniswami, "A novel fuzzy rule based system for assessment of ground water portability: a case study in South India", Magnesium (Mg), 30 (2013), pp. 3541.

[7] Lamiaa F. Ibrahim, Reem Albatati, Samah Batweel, Rudainah Shilli, Mai Bakeer, Tsneem Abo Al Laban, "Safety of Natural Disasters", Design, User Experience, and Usability. User Experience in Novel Technological Environments, Book Series: Lecture Notes in Computer science, Volume 8014, 2013, pp 85-94, Springer Berlin Heidelberg.

[8] Nabil R. Adam, Basit Shafi q, Robin Staffin, "Spatial Computing and Social Media in the Context of Disaster Management", Intelligent Systems, IEEE, vol. 27, no. 6, pp. 90-96, Nov.-Dec. 2012 doi: 10.1109/MIS.2012.113.

[9] Gaia

https://play google.com/store/apps/details?id=com.tralbehind.andr aiagps.pro\&feature=search_result\#?t=W251bGwsMSwxLDEsImNvbS5 0cmFpbGJlaGluZC5hbmRyb2lkLmdhaWFncHMucHJvIl0

[10] ubAlert- Disaster Alert Network. [Online]. http://itunes.apple.com/us/app/ubalert-disaster-alertnetwork/id455647397?mt=8

[11] Disaster Alert. [Online]. https://play.google.com/store/apps/details?id=disasterAlert.PDC
[12] Rehka Jadhav, Jwalant Patel, Darshan Jain, Suyash Phadhtare, "Emergency Management System Using Android Application", International Journal of Computer Science and Information Technologies, Vol. 5 (3), 2014, 2803-2805.

[13] Jungki Lee; Niko, D.L.; Hyunsuk Hwang; ManGon Park; Changsoo Kim, "A GIS-based Design for a Smartphone Disaster Information Service Application," Computers, Networks, Systems and Industrial Engineering (CNSI), 2011 First ACIS/JNU International Conference on, vol., no., pp.338,341, 23-25 May 2011 doi: $10.1109 /$ CNSI.2011.13

[14] (2016) http://globaltechconsultants.org/?q=content/phpmyadmin

[15] Wikipedia (2012), The Free encyclopedia website, AutoCAD [Online] available from http://en.wikipedia.org/wiki/AutoCAD

[16] (2006) EB2A Internet Services. [Online]. https://www.eb2a.com/en/

[17] Sandip Patil. (2009, December ) Android Project structure. [Online]. http://www.mobisoftinfotech.com/blog/android/android-projectstructure/

[18] (2012, December) Intent Structure. [Online] http://developer.android.com/reference/android/content/Intent.html

[19] (2012, December) Launcher Icons. [Online]. http://developer.android.com/guide/practices/ui_guidelines/icon_design_ launcher.html

[20] (2012, December) Obtaining a Google Maps Android v1 API Key. [Online]. https://developers.google.com/maps/documentation/android/v1/mapkey? $\mathrm{hl}=\mathrm{ar}-\mathrm{SA}$

[21] (2012, December) Knowledge base. [Online]. http://en.wikipedia.org/wiki/Knowledge_base

[22] Disaster Alert, (2015, Jan.), [online]. https://play.google.com/store/apps/details?id=disasterAlert.PDC

[23] Disaster Message Board Service, (2015, Jan.) [online]. https://www.nttdocomo.co.jp/english/info/disaster/disaster_board/

TABLE I. COMPARISON BETWEEN SYSTEMS

\begin{tabular}{|l|l|l|l|l|}
\hline & iCG & DSRG & Disaster Alert[22] & $\begin{array}{l}\text { Disaster Message } \\
\text { Board [23] }\end{array}$ \\
\hline Indoor & Yes & No & No & No \\
\hline Guide user & Yes & Yes & No & No \\
\hline Network type & $\begin{array}{l}\text { 3G or WIFI or } \\
\text { Mobile network }\end{array}$ & $\begin{array}{l}\text { 3G or WIFI or } \\
\text { Mobile network }\end{array}$ & Mobile network & Mobile network \\
\hline Reliability & High & High & Low & Low \\
\hline $\begin{array}{l}\text { Update } \\
\text { Information }\end{array}$ & Yes & Yes & Yes & No \\
\hline
\end{tabular}

